\title{
Randomized Permutation Routing in Multi- hop Ad Hoc Networks with Unknown Destinations
}

\author{
Djibo Karimou and Jean Frédéric Myoupo \\ LaRIA, CNRS, FRE 2733, \\ Université de Picardie Jules-Verne \\ 33, tue Saint Leu, 80039 Amiens, France \\ E-mail:\{karimou, myoupo\}@laria.u-picardie.fr
}

\begin{abstract}
A large variety of permutation routing protocols in a single-hop Network are known to day. Since they are single hop, there is always a wireless path connecting two nodes. One way to solve this problem in a multiple hop environment is to partition nodes into clusters, where a node in each cluster called clusterhead is responsible for the routing service. In this paper, we propose a clustering mechanism to perform permutation routing in multi-hop ad hoc Networks having $p$ stations and in which $n$ data items are saved. We first develop a clustering algorithm to partition stations into clusters. Secondly, we run a locally permutation routing to broadcast items to their local destinations in each group. Finally we use a multicast procedure to transmit outgoing items to their final cluster destination. We show that the approach of this paper can solve the permutation problem in a multi hop ad hoc network in $\left(\frac{6}{q}+13\right) n+O\left(q^{\frac{4}{3}} \log ^{\frac{10}{3}} q\right)+D_{b}-1$ broadcast rounds in the worst case
\end{abstract}

with the probability at least $1-\frac{1}{p}$ whenever $q \leq \frac{p}{3 \log p}$. Where $D_{b}$ is the blocking diameter, $q$ is the number of clusters of the network. In our knowledge, it is the first algorithm for permutation routing in multi-hop ad hoc networks.

\section{Introduction}

In an environment where there is no communication infrastructure, wireless mobile users may still be able to communicate through the formation of an ad hoc network. Mobile ad-hoc networks are formed by a collection of mobile wireless nodes (stations) which can communicate with each other and dynamically self organize without any static network interaction. Each mobile host has the capability to communicate directly with another mobile host in its vicinity via a channel of transmission. It can also send packets destined for other nodes.

Please use the following format when citing this chapter:

Karimou, D., Myoupo, J.F., 2006, in International Federation for Information Processing (IFIP), Volume 212, Ad-Hoc Networking, ed. Al Agha, K., (Boston: Springer), pp. 47-59. 
Communication links are formed and disappear as nodes come into and go out of each other communication range. Such networks have many practical applications, including home networking, search-and-rescue, and military operations. There are two types of ad hoc networks: Single hop ad hoc network in which each station can transmit or communicate directly with each other station. All the stations use the same channel to communicate, and the message broadcasted by one of the stations on the common channel is simultaneously received by all other stations. In the multi hop ad hoc networks intermediate nodes are used to route message from the source to the destination. In this paper only to multi-hop ad hoc networks are considered.

Permutation Problem: Consider a MANET ( $\mathrm{n}, \mathrm{p}$ ) of $p$ stations with $n$ items pretitled on it. Each item has a unique destination which is one of the $p$ stations. Each item has a unique destination which is one of the $\mathrm{p}$ stations. Each station has a local memory of size $p / n$ in which $n / p$ items are stored.

It is important to note that in general, some of the $n / p$ items stored in the station, say $i$, have not $i$ as destination station. And even, it can happen that none of these $n / p$ items belongs to it. In the other hand, the situation in which initially all items in $i$ belong to $i$ can also occur. The permutation routing problem is to route the items in such a way that for all $i, 1 \leq i \leq p$, station $i$ contains all its own items.

A large variety of permutation routing protocols in a single-hop Network are known to day. These permutation routing protocols assume that the network are a single Hop Ad-Hoc Network, hence there is always a path connected by wireless links between a source and the destination. However, these varieties of methods are not adapted in the case of multi-hop Ad Hoc Networks. One way to solve this problem is to partition nodes into clusters where principal node in each cluster, called clusterhead, is responsible for routing items. In this paper, we suggest a combination of methods which guarantee a permutation routing protocol in multihop ad hoc network.

\subsection{Known Results}

The number of studies specifically targeted to permutation routing in single hop mobile ad hoc networks has grown significantly:

It is shown in [9] that the permutation routing of $n$ items pretitled on mobile ad hoc network of $\mathrm{p}$ stations ( $\mathrm{p}$ known) and $\mathrm{k}$ channels (MANET(n, $\mathrm{p}, \mathrm{k})$ for short) with $k<p$, can be carried out in $2 n / p+k-1$ broadcast rounds if $k \leq \sqrt{ } p$ and if each station has a $O(n / k)$-memory locations. If $k \leq \sqrt{(p / 2)}$ and if each station has a $O(n / p)$ memory locations, the permutations of these $n$ pretitled items can be done also in $2 n / p+k-1$ broadcast rounds. In [8], we solve the problem showing how the restriction can be left. More precisely, we show that the permutation routing problem can be solved on it in $O(p / \ln 2)+(2 / k+1) n+k-1$ broadcast rounds in the worst case. It can be solved in $O(p / \ln 2)+(2 / k) n+k+1$ broadcast rounds in the better case, without any restriction on $\mathrm{k}$. In [4], we solve the problem with $n$ unknown.

Recently, Datta in [2] presented a fault toler ant permutation routing protocol of $n$ items pretitled on mobile Ad-hoc network of $p$ stations and $k$ channels MANET( $n, p$, k) for short. He solved the fault-tolerant permutation routing problem in $2 n / k+($ $p / k)^{2}+3 p / k+2 k^{2}-1$ slots and each station needs to be awake for at most $\left(4 n f_{i}\right) / p+$ $2 n / p+3 p / k+k^{2}+p / 2 k+p / k+4 k$ slots, where $\mathrm{f}_{\mathrm{i}}$ is the number of faulty stations in 
a group of $\mathrm{p}$ stations. He also assumed that $k \leq \sqrt{ }(p / 2)$ and in the presence of faulty stations some data items are lost. We came out with our work in [5] presenting a fault tolerant protocol which avoids the lost of items. The authors in [10] came out with the first energy-efficient permutation routing. A more efficient energy-efficient permutation routing protocol appeared in [3].

Finally, in $[2,9,10]$ it is assumed that a station knows the destination stations of each data item it holds. And that even after the partition in groups, a station knows the destination group of each item of its local memory.

\subsection{Our Results}

We consider a MANET(n, p) with $n$ items, $p$ stations. A station does not know the destination stations of data items it holds. We develop a protocol which first partitions the stations in clusters. Next we show that the permutation routing problem in multi-hop ad hoc network can be solved on it in $\left.\frac{(-6}{q}+13\right) n+O\left(q^{\frac{4}{3}} \log ^{\frac{10}{3}} q\right)+D_{b}-1$ broadcast rounds in the worst case with the probability at least $1-\frac{1}{p}$ whenever $q \leq \frac{p}{3 \log p}$. Where $q$ is the number of clusters of the network. To the best of our knowledge, this is the first permutation routing algorithm in a multi-hop ad hoc network.

The rest of this work is organized as follows: Some definitions and the environment considered in this work are presented in section 2 . In section 3 we introduce some probability tools which are essential for the rest of the paper. The phases of permutation routing are presented in section 5 . A conclusion ends the paper.

\section{Preliminaries}

An Ad Hoc Network is a set of $n$ radio transceivers or stations which can transmit and/or receive messages from each other. The time is assumed to be slotted and all stations have a local clock that keeps synchronous time. In any time slot, a station can tune into one channel and/or broadcast on at most one channel. A broadcast operation involves a data packet whose length is such that the broadcast operation can be completed within one time slot. So, in the MANET with Collision detection (CD for short), the status of an $n$-station MANET channel is:

-NULL: if no station broadcasts on the channel in the current slot,

-SINGLE: if exactly one station broadcasts on the channel and

-COLLISION: if two or more stations broadcast on the channel in the current time slot.

Also, all the communications are performed at time slots boundaries i.e. the duration operation is assumed to be sufficiently short. 
(i) . All communication is over wireless links. A wireless link can be established between a pair of nodes only if they were within wireless range of each other. Two nodes that have a wireless link, will be said to be 1 wireless hop away from each other. There are also called neighbours.

(ii) . Each station belonging to a cluster is a resident of cluster. Hence, this station may in a given time unit, broadcast a message to its neighbours.

\section{Definition 1}

Let us consider $p$ stations $1,2, \ldots, p$ which communicate in a multi-hop ad hoc Network MANET ( $n, p)$. We suppose that we have $n$ items in the system. Then each station of a MANET $(\mathrm{n}, \mathrm{p})$ is assumed to have a local memory of size at least $O(n)$.

\subsection{Problem Description}

We suppose that the $\mathrm{n}$ items denoted $a_{1}, a_{2}, \ldots, a_{n}$ are pretitled on a MANET(n, p) such that for every $i, 1 \leq i \leq p$, station i stores the $\mathrm{n} / \mathrm{p}$ items $a_{(i-1) \cdot n / p+1}, a_{(i-1), n / p+2}, \ldots$. $a_{(i) . n / p .}$ Each item has a unique destination station. It is important to note that hereafter a station does not know the destination of items it holds. It is assumed that the items are encoded in such a way that only the destination stations can recognize its own data items. A station which has received and cracked an encoded data item is capable to recognize that it is its item. If it is not its own item, then it is impossible for it to know the destination station to whom belongs the item. For every v, $1 \leq v \leq p$, let $h_{v}$ be the set of items whose destination is station v.

The permutation routing problem with unknown destinations is to route items in such a way that for all $v, 1 \leq v \leq p$, station $\mathrm{v}$ contains all items in $\mathrm{h}_{\mathrm{v}}$. Consequently, each $\mathrm{h}_{\mathrm{v}}$ must contain exactly $\frac{n}{-}$ items (see figure 1 for an example).

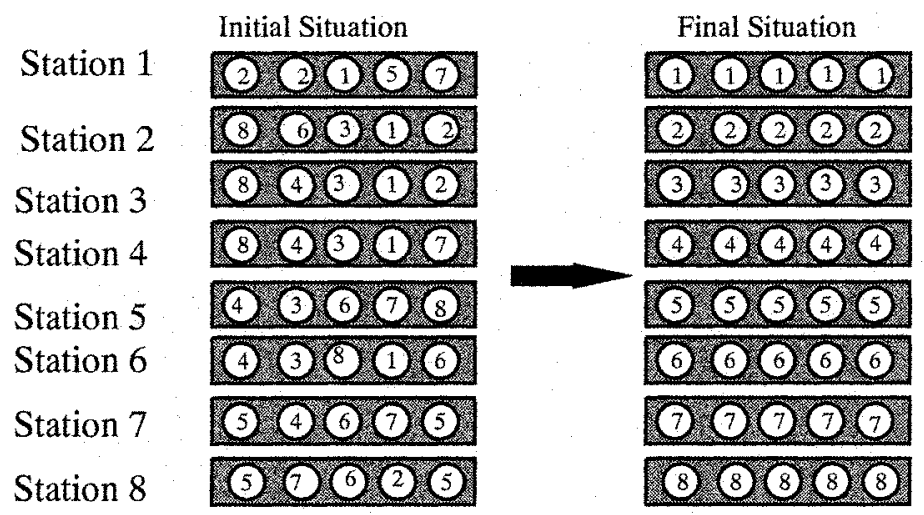

Figure 1: Permutation routing

It is important to note that hereafter a station does not know the destinations of items it holds. It is assumed that the items are encoded in such a way that only the destination stations can recognize its own data items. A station which has received 
and cracked an encoded data item is capable to recognize that it is its item. If it is not its own item, then it is impossible for it to know the destination station to which owns the item.

\section{Some probability tools}

Throughout this paper $\operatorname{Pr}[\mathrm{E}]$ will denote the probability of event $\mathrm{E}$. For a random variable $\mathrm{X}, \mathrm{E}[\mathrm{X}]$ denotes the expected value of $\mathrm{X}$. Let $\mathrm{X}$ be a random variable denoting the number of successes in $\mathrm{p}$ independent Bernouilli trials with parameters $v$ and $1-v$. It is well known that $\mathrm{X}$ has a binomial distribution and that for every $\mathbf{r}$, $(0 \leq \mathrm{r} \leq \mathrm{p})$,

$$
\operatorname{Pr}[X=r]=\left(\begin{array}{l}
p \\
r
\end{array}\right) v^{r}(1-v)^{p-r}
$$

The expected value of $X$ is then given by

$$
E[X]=\sum_{r=0}^{p} r \cdot \operatorname{Pr}[X=r]=p v
$$

To analyse the tail of the binomial distribution, the following estimates, commonly referred to as Chernoff bounds [7], are considered:

$$
\begin{aligned}
& \operatorname{Pr}[X \leq(1-\varepsilon) E[X]] \leq e^{-\frac{\varepsilon^{2}}{2} E[X]}, 0 \leq \varepsilon \leq 1 \\
& \operatorname{Pr}[X \geq(1+\varepsilon) E[X]] \leq e^{-\frac{\varepsilon^{2}}{3} E[X]}, 0 \leq \varepsilon \leq 1
\end{aligned}
$$

\section{Permutation routing protocol}

Our Approach to provide permutation routing in multi-hop Ad Hoc network consists of the following four steps:

1. Partitioning stations into clusters. This method of clustering is based on [1].

2. Local cluster broadcasting: In each cluster, we apply a method of local permutation routing within each cluster. Data items which belong to a resident of a cluster are locally broadcasted to their destinations.

3. Extra-cluster broadcasting: it allows broadcastings of items which were recorded not belong to local stations, (say outgoing items) to their final cluster destinations.

4. Ordering the cluster for broadcasting: after this phase each clusterhead knows when it is going to carry out broadcastings.

5. Local cluster re-broadcasting. Again, we run a local broadcast in each cluster to transmit the new arrived items to their final destinations. 


\section{step 1: Clustering procedure}

The main task of this section is to partition multi-hop network into clusters, so that local broadcastings can be accomplished in parallel in clusters. The goal of the clustering algorithm is to partition network into several clusters. Assume that we have undirected graph $\mathrm{G}(\mathrm{V}, \mathrm{E})$ representing a communication network where $\mathrm{V}$ is the set of nodes (mobiles) $\mathrm{V}=\left\{v_{1}, v_{2}, \ldots v_{p}\right\},\{|V|\}$, and $\mathrm{E}$ is the set of edges representing communication links. We consider that each node $v_{i}$ has a positive weight $\left(w_{i}\right)$. For two nodes $v_{i}, v_{j}, w_{i} \neq w_{j}$. for $i \neq j$. Also, we assume that for two nodes $v i, v_{j}$, edges $e_{i j}=e_{j i}$ since the graph is symmetric. The clusters are denoted by $\mathrm{C}_{i}$ for $1 \leq i \leq q$ and cluster heads $\mathrm{CH}_{\mathrm{i}}$ for $1 \leq i \leq q$. The clustering divides $\mathrm{V}$ into a collection of $k$ subsets $V_{1}, V_{2}, \ldots V_{k}$ where $V=U_{i=1} V_{i}$; such that each subsets $V_{i}$ induces a connected sub-graph of $\mathrm{G}$.

In some case, sub-graphs can overlaps or not, and each subset is considered as a cluster. This approach is based on [1] in which Basagni proposed to use node's weights instead of lowest ID or nodes degrees in cluster head decisions.

We make the following assumptions underlying the construction of the clusters.

- Every node knows its degree, its weight, the degrees and the weights of its neighbours

- At the end of the clustering each cluster head knows the weight of the others cluster heads.

This clustering involves two phases:

Phase 1: Choice of cluster head. Each cluster is formed with a cluster head and some neighbour nodes called ordinary nodes. The choice of the clus ter head is based on the degree associated to each node. In the case of equality, the bigger the weight of the node is, the better that node is for the role of cluster head. Then nodes with highest degrees initiate the clustering process by flooding [11] request to be cluster head to all its neighbours nodes. Hence, all nodes which degree is bigger than those of all its neighbours broadcasts its decision to be a cluster head. If a node knows one of its neighbours with highest degree than itself, it resigns and adopts that node as a cluster head. A node $v_{i}$ becomes a cluster head if at least one of the following propriety is satisfied:

$-v_{i}$ has the highest degree among all nodes within 1 wireless hop from him, or

$-v_{i}$ has not the highest degree among its neighbours but all its 1 wireless hop nodes belong to other clusters.

Phase 2: Choice of ordinary nodes. For a node which degree is smaller than the ones of its neighbours, two cases can happen:

- if this node has one neighbour who is a cluster head, in this case, it moves directly to belong to the cluster formed by this neighbour.

- if its neighbour with highest degree belongs to another cluster then two cases are possible: if there is another cluster head among its neighbours, it moves or becomes a member of this cluster, else it creates a new cluster.

We assume that at the end, our approach yields k clusters. 

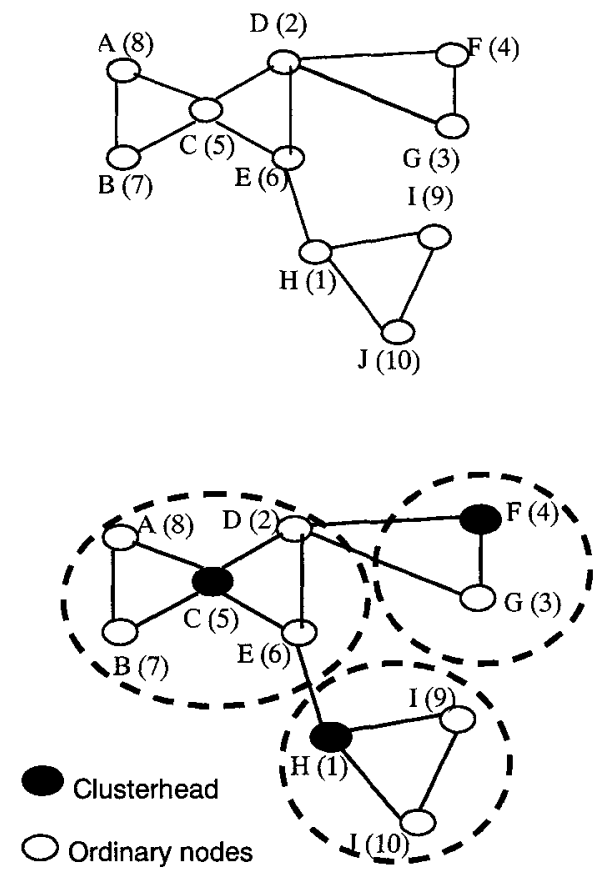

Figure 3 : clustering algorithm

For example, the clustering algorithm in figure 2 produces clusters as indicated in figure 3 . The algorithm creates a non-overlapping clusters in which each node selects one of its several neighbouring cluster head. At the end of the clustering the following proprieties are satisfied:

- Entire network is divided into clusters. Each node belongs to exactly one cluster and a node is either a cluster head or ordinary node which is directly connected to one of the cluster head among its neighbours with highest degree.

- All clusters have a radius of size two. A node either becomes a clusterhead, or is at most 1 wireless hop away from its clusterhead.

- Also at the end of clustering, the number of nodes $\left(p_{i}\right)$ in each cluster is known by the cluster head. Nodes at the periphery of a cluster called gateway nodes are known from the two cluster heads. Gateway nodes are the nodes at the border of a cluster and typically communicate with gateway nodes of the other clusters. They fetch into their cluster items broadcasted by cluster heads of the other clusters. And also, they are the nodes that have the responsibility of routing items which were recorded not belonging to any local node to the other clusters.

- Every ordinary node has at least a cluster head as a neighbour and belongs to a cluster formed by its neighbours with highest degree.

- No two cluster heads can be neighbours and also ordinary nodes are 1-hop to a cluster head. 
Our algorithm is based on the method of Basagni [1], certainly with a slight (we take the degree into account) modification but, which does not influence the complexity of the algorithm. Hence the worst complexity of the algorithm is $D_{b}-1$ steps with $D_{b} \leq p-1$ (where $D_{b}$ is the blocking Diameter. (see [1] for more detail). Although the complexity of the algorithm is proved to be bounded by a network parameter that depends on the possibly changing topology of the mobile ad hoc network, rather $\mathrm{p}$, in some case the possibility to have $\mathrm{p}$ can occur. Hence, with a multi-hop Ad Hoc network, with p station, the clustering procedure needs in average $p$ broadcast rounds.

We now assume that there are $q$ clusters. Fix a cluster and let $\mathrm{X}$ be the random variable denoting the number of stations that selected that cluster. Thus we have $E[X]=\frac{p}{q}$. By using the Chernoff bound in (4) with $\varepsilon=1$, we can bound the probability $\operatorname{Pr}\left[X \geq \frac{2 p}{q}\right]$ that the cluster have $\frac{2 p}{q}$ or more stations as follows:

$$
\operatorname{Pr}\left[X \geq \frac{2 p}{q}\right] \leq e^{\frac{-2 p}{3 q}} .
$$

Thus, the probability that some cluster has at least $\frac{2 p}{q}$ stations is less than $k \cdot e^{\frac{-2 p}{3 q}}$. It follows that, with the probability at least $1-k \cdot e^{\frac{-2 p}{3 q}}$, all the clusters have fewer than $\frac{2 p}{q}$ stations. Hereafter, we assume that each of the $\mathrm{k}$ clusters contains in maximum $\frac{2 p}{q}$ stations.

\section{Step 2. Local Cluster Broadcastings}

We assume that the multi-hop ad hoc networks is partitioned into $\mathrm{k}$ clusters, $\mathrm{C}_{1}$, $\mathrm{C}_{2} \ldots \mathrm{C}_{\mathrm{k}}$. Cluster heads are known, and also gateway nodes are known in each cluster. We consider that cluster head $\left(\mathrm{CH}_{\mathrm{i}}\right.$ is vested with the responsibility to perform local broadcast in cluster $\mathrm{C}_{\mathbf{i}}$. All local stations are at 1 wireless hop of their clusterheads. Each node has a local memory of size $O(n)$.

\section{Assumption 4.1}

Hereafter we assume that we are always in the worst case, i.e. non data item in a cluster $C_{i}$ belongs to a station in $C_{i}$.

After this clarification, it is important to note that here broadcastings are local in each cluster. Every data item broadcasted is accompanied with a bit 0 to mean that the broadcast is local. Therefore cluster gateway may not broadcast data items out of the cluster. We only focus on the routing that takes place in cluster $C_{1}$, the local broadcasts on all other groups are similar. The cluster head knows the weight of its residents. With the help of these weights, the cluster head knows the turn of broadcasting of each resident, from the smaller weight to the bigger. The cluster invited each of its residents to broadcast to him all the data items which are not their own data items. Hence, each node broadcasts in its turn according to its weights. It 
begins with the nodes with the highest weight, followed by the next with the highest weight, and so on (figure 4).

phase $i$ : First, one after another, from station with highest weight to station with least weight, in this order, stations broadcast items which do not belong to them, to their cluster heads, one by one. The cluster head $\mathrm{CH}_{1}$ copies these items to its local

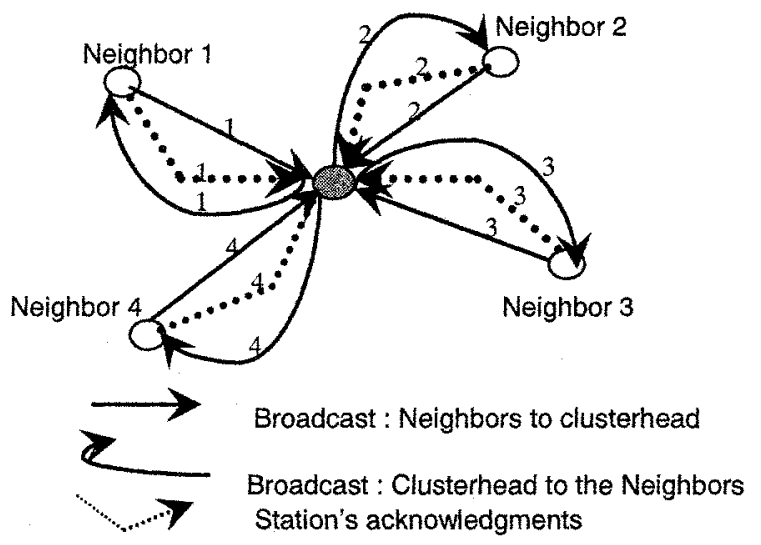

Figure 4 : Local Cluster Broadcast

memory and keeps those which are its own items. This sub-phase needs $\frac{2 p}{q} \times \frac{n}{p}=\frac{2 n}{q}$ broadcast rounds in the worst case in cluster $\mathrm{C}_{\mathrm{i}}$ )

phase ii: The cluster head broadcasts all data items (except those which are its own) it receives to its neighbours simultaneously, one by one by multiplexing. Assume that the cluster head $\mathrm{CH}_{\mathrm{i}}$ is carrying out the local broadcasting to a station $\mathrm{v}$, two cases can occur:

- if a item belongs to $\mathrm{v}$, then $\mathrm{v}$ copies this items to its local memory. In the next slot $\mathrm{v}$ broadcasts a positive acknowledgment to $\mathrm{CH}_{1}$ to inform that it is its item.

- if a item does not belongs to $\mathrm{v}$, then no positive acknowledgment will be send. After a timeout it is recorded not belonging to any resident of the cluster $\mathrm{C}_{1}$, it is a outgoing item. It is then saved by the cluster head as outgoing item.

So, at the end of the step, all the items which were recorded not belong to any node in $\mathrm{C}_{1}$ are identified and stored by the cluster head $\mathrm{CH}_{1}$ as outgoing items. Since, each station in $\mathrm{C}_{1}$ has $\frac{n}{p}$ items, this step needs $2 \frac{n}{p} \times \frac{2 p}{q}=\frac{4 n}{q}$ broadcast rounds (it is clear that the number of nodes in cluster $\mathrm{C}_{1}$ is $\frac{2 p}{q}$ ). Therefore $\frac{4 n}{q}$ rounds are necessary in the worst case to carry out this step, $1 . i \leq q$. Note that the worst case is the one in which no node detains any of its own data items. 
Then $\frac{6 n}{q}$ broadcast rounds are necessary to carry out step 2 .

\section{Step 3: Ordering the clusters for broadcastings: gossiping}

It is important to know the order in which the clusters are going to broadcast. The clusters broadcast simultaneously theirs weights to each other. Since all weights are different, a cluster after receiving all weights knows exactly the time when it will start broadcasting. We assume that that we are in the worse case where none data item in a cluster does not belong to a node in the cluster. According to [6] this step needs $O\left(q^{\frac{4}{3}} \log { }^{\frac{10}{3}} q\right)$ broadcast rounds.

\section{Step 4: Out-going data items Broadcastings}

This step is a procedure which helps to move items which are recorded not to belonging to any local station to their final destination into the other clusters. Here, every data item broadcasted is accompanied with a bit 1 to mean that the broadcast is extra cluster. In fact the network is reduced to the one which nodes are just clusters. Therefore a data item needs 6 broadcast rounds to leave a cluster: one from a gateway node to the clusterhead, one from the clusterhead to a gateway node, one to leave the cluster, and when the packet reaches the next cluster, it broadcasts an acknowledgement packet to the previous cluster which has sent the packet. Consequently a cluster broadcast a data item every 6 slots (we recall that the time is slotted). On the level of each cluster $\mathrm{C}_{\mathrm{i}}$, supervised by its clusterhead $\mathrm{CH}_{\mathrm{i}}, \mathrm{CH}_{\mathrm{i}}$ sends one by one each items which were recorded not to belonging to any station in $C_{i}$ (outgoings items) to the other clusters $C_{j}(i \neq j)$. It should be noted that the gateway nodes serve only as routers to relay items towards the clusterheads nodes to which they are connected.

Again, we only focus on the multicast from $C_{1}$. The clusterhead in $C_{1}$ broadcasts first, followed by those in $C_{2}$, next $C_{3}$ until $C_{k}$ (ordered by their weights). The clusterhead of $\mathrm{C}_{1}\left(\mathrm{CH}_{\mathrm{i}}\right)$ sends one by one, every 6 slots, each outgoing item to all the destinations, among $\mathrm{C}_{2}, \mathrm{C}_{3} \ldots \mathrm{C}_{\mathrm{k}}$, to which it is connected, via its gateway nodes. Each clusterhead copies received items in its local memory, i.e. the $\mathbf{n}$ data items. After $\mathrm{C}_{1}$ has broadcasted all its outgoing items, it broadcasts an information to the next cluster, say $\mathrm{C}_{2}$ to mean that it is its turn to carry out multicast broadcasting.

Now to evaluate upper bound of the running time of this step, note that $\mathrm{k}$ is the diameter of the network formed by clusters (here each cluster is considered as a simple node). A cluster needs $6\left(\frac{2 p}{q} \times \frac{n}{p}\right)=\frac{12 n}{q}$ broadcast rounds are used by the nodes in cluster $\mathrm{C}_{\mathrm{i}}$ to forward their outgoing items. In total this step takes $q\left(\frac{12 n}{q}\right)=12 n$ where $\mathrm{k}$ is the number of clusters. As we always assume that we are in the worst case, this step brings into $C_{i}$ all data items which destination stations are in a cluster $\mathrm{C}_{\mathrm{i}}$. 


\section{Step 5 : Local cluster re-broadcastings}

This step is similar to step2. Each cluster head broadcasts the data items that were stored during step4 to all its neighbours (ordinary nodes). The procedure is carried out in parallel in clusters. One by one, the data items are simultaneously broadcasted, by multiplexing, from the cluster heads to its neighbours. At the end of this step, ach ordinary node recovers all its own items. The reader should not have difficulty to confirm that this procedure in cluster $\mathrm{C}_{\mathrm{i}}$, needs $n$ broadcast rounds in the worst case.

\section{Theorem 4. 1}

Let $p$ mobile stations in a Multi-hop Ad-Hoc network $(n, p)$, with p stations and $n$ items pretitled on it. The permutation routing problem can be solved in $\left(\frac{6}{q}+13\right) n+O\left(q^{\frac{4}{3}} \log ^{\frac{10}{3}} q\right)+D_{b}-1$ broadcast rounds in the worst case with the $q$

probability at least $1-q . e^{\frac{-2 p}{3 k}}$. Where $k$ is the number of clusters, $D_{b}$ is the blocking diameter.

\section{Proof:}

i) Step 1 of the algorithm need $D_{b}-1$ steps with $D_{b} \leq p-1$

ii.) In step $2, \frac{6 n}{q}$ broadcast rounds are necessary for its execution in the worst case

iii.) Step 3 needs $O\left(q^{\frac{4}{3}} \log ^{\frac{10}{3}} q\right)$ broadcast rounds

iv.) Step 4 runs in $12 n$ broadcast rounds.

v.) Step 5 runs in $n$ broadcast rounds.

Therefore the number of broadcast rounds need by the protocol results of the summation of the running time of the five steps. Therefore, our protocol runs in $\left(\frac{6}{q}+13\right) n+O\left(q^{\frac{4}{3}} \log \frac{10}{3} q\right)+\mathrm{D}_{\mathrm{b}}-1$ broadcast rounds. Where is $\mathrm{D}_{\mathrm{b}}$ is the blocking Diameter $\left(D_{b} \leq p-1\right)$.

Remark4.1. If we assume that $q \leq \frac{p}{3 \log p}$, then one has $q e^{\frac{-2 p}{3 q}} \leq \frac{p}{3 \log p} \cdot e^{-2 \log p}<\frac{1}{p} \cdot$ Consequently, we have the following:

Corollary 4. 1. Let p mobile stations in a Multi-hop Ad-Hoc network (n, p),with $p$ stations and $n$ items pretitled on it. The permutation routing problem can be solved 


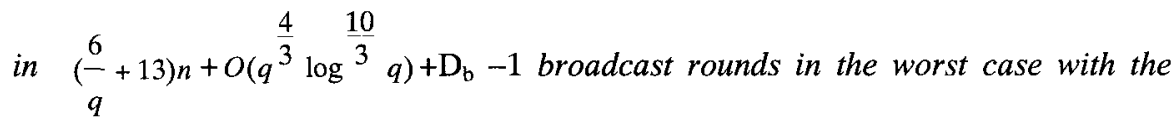
probability at least $1-\frac{1}{p}$ whenever $q \leq \frac{p}{3 \log p}$.

\section{Conclusion}

In this paper, we have considered a Multihop ad hoc network with $\mathrm{n}$ items, $\mathrm{p}$ stations, with $\mathrm{p}$ unknown. First, we present a clustering approach to partition stations into $\mathrm{k}$ groups. Finally, we show that the permutation routing problem can be solved in $\left(\frac{6}{q}+13\right) n+O\left(q^{\frac{4}{3}} \log ^{\frac{10}{3}} q\right)+D_{b}-1$ broadcast rounds in the worst case with the probability at least $1-\frac{1}{p}$ whenever $q \leq \frac{p}{3 \log p}$. To the best of our knowledge, this is the first algorithm on the permutation routing in a multi-hop ad hoc networks. A slight modification of our protocol as follows can help to manage simultaneous broadcastings to the clusterhead: a gateway station can broadcast to the clusterhead only if he is invited to do so by the clusterhead. Our approach assumes that the $q$ most weighted stations are first thrown in the area. Then each of the p-q remained stations chooses at random to be a neighbour of one of the q clusterheads.

However some open problems remains. The derivation from the idea of this paper of a fault tolerant algorithm, which guarantees the delivery of data items to non faulty nodes, is to be investigated. Also, the construction of an energy-efficient permutation routing protocol for multi-hop ad hoc network is a challenge.

\section{References}

[1] S. Basagni. Distributed clustering for multihop wireless networks. In $A$. Annamalai and C. Tellambura, Proceedings of the IEEE International Symposium on Wireless Communications (ISWC'99), pp. 41-42, June 3-4 1999.

[2] A. Datta. Fault-tolerant and Energy-efficient Permutation Routing Protocol for Wireless Networks. $17^{\text {th }}$ IEEE Intern. Parallel and Distributed Processing Symposium (IPDPS'03), Nice, France, 2003.

[3] Amitava Datta, Albert Y. Zomaya: An Energy-Efficient Permutation Routing Protocol for Single-Hop Radio Networks. IEEE Trans. Parallel Distrib. Syst. 15(4): 331-338 (2004)

[4] D. Karimou, J. F. Myoupo. An Application of an Initialization Protocol to Permutation Routing in a Single-hop Mobile Ad-Hoc Networks. The journal of Supercomputing vol. 31, p. 215-226, 2005.

[5] D. Karimou and J.F. Myoupo. A Fault Tolerant Permutation Routing Algorithm in Mobile Ad Hoc Networks. International Conference on Networks (ICN'05), Part II, LNCS 3421, pp.107-115, 2005. 
[6] L. Gasieniec, T. Radzik and Q. Xin. Faster deterministic gossiping in ad hoc radio networks. Proc. $9^{\text {th }}$ Scandinavian Workshop on Algorithm Theory, SWAT 2004, Humlebaek, Danmark, LNCS 3111, pp. 397-407.

[7] R. Motwani and P. Raghavan. Randomized Algorithms. Cambridge University Press, 1995.

[8] J. F. Myoupo. Concurrent Broadcasts-Based Permutation Routing algorithms in Radio Networks. IEEE Symposium on Computers and Communications, (ISCC'03), Antalya, Turkey, 2003.

[9] K. Nakano, S. Olariu and J.L. Schwing. Broadcast-Efficient protocols for Mobile Radio Networks. IEEE Trans. Parallel Distr. Syst. vol.10, pp.12, 1276-1289, 1999.

[10] Koji Nakano, Stephan Olariu, Albert Y. Zomaya: Energy-Efficient Permutation Routing in Radio Networks. IEEE Trans. Parallel Distrib. Syst. 12(6): 544-557 (2001)

[11] Y.C. Tseng, S-Y Ni, Y-S Chen and J.-P Sheu. The Broadcast Storm Problem in a Mobile Ad Hoc Network. ACM Wireless Networks 8, 153-167, 2002 